\title{
Monitorización terapéutica de antimicrobianos en pediatría. Revisión de la experiencia latinoamericana
}

\author{
Brenda Zylbersztajn, Marlon Barraza, Juan P. Torres y Jorge Morales
}

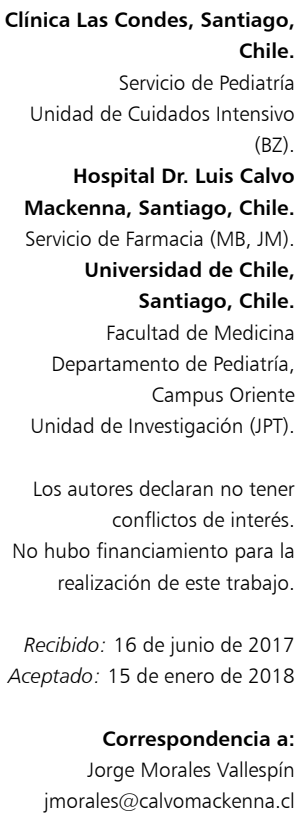

\section{Introducción}

M uchos fármacos utilizados en la práctica pediátrica tienen regímenes de dosificación basados en la evidencia. No obstante, una revisión reciente muestra que 13 a $30 \%$ de los medicamentos utilizados en atención primaria y 49 a $87 \%$ en los hospitales se prescriben off label en pediatría (sin aprobación de la indicación) ${ }^{1}$. En términos de la dosificación, en niños suele ser empírica, basada en el peso de cada paciente, de una manera lineal y derivada de una dosis extrapolada de adultos. Utilizando dosis por $\mathrm{kg}$ de peso del paciente, la dosificación puede conducir a una sobredosificación o una subdosificación, especialmente en grupos de edad específicos como neonatos o pacientes pediátricos en shock séptico, generando la introducción de toxicidad o falta de eficacia en la farmacoterapia ${ }^{2}$. Afortunadamente, para muchas infecciones, una dosificación exacta puede no ser siempre crítica, ya sea por amplio rango terapéutico del fármaco escogido y el riesgo mínimo de toxicidad resultante o porque el patógeno es altamente sensible a concentraciones bajas de fármaco. Esta última característica se relaciona con la farmacodinamia (FD), que describe la relación de la dosis observada en el cuerpo y el poder bactericida y/o la capacidad de inhibir el crecimiento del patógeno. Para establecer esta relación, la concentración inhibitoria mínima (CIM) de un patógeno particular, es el factor clave que se utiliza para definir objetivos farmacocinéticos/farmacodinámicos (FC/FD) para agentes anti-infecciosos.
Tradicionalmente, la mayoría de los antimicrobianos pueden categorizarse en tres grupos dependiendo de sus propiedades FC/FD, ellas son: tiempo que la concentración plasmática del antimicrobiano permanece sobre la CIM ( T > CIM), área bajo la curva $(\mathrm{ABC})$ durante la cual la exposición del fármaco está sobre la CIM (AUC/ CIM) y concentración plasmática máxima sobre la CIM $(\mathrm{Cmax} / \mathrm{CIM})^{3}$.

Las decisiones sobre los fármacos óptimos utilizados para tratar las infecciones se basan en numerosas suposiciones, incluyendo que una determinada dosis dará lugar a una concentración del fármaco en el cuerpo (FC) y que esta concentración será suficiente para matar y/o inhibir el crecimiento del patógeno (FD). Sin embargo, en ciertas situaciones clínicas tales suposiciones son inexactas y se ha demostrado que el resultado mejora cuando se miden las concentraciones del fármaco como parte de la monitorización terapéutica (MT). La MT se define como la medición de concentraciones del fármaco en el suero o plasma y posterior ajuste de la dosis para el individuo, según los conceptos FC/FD. Se puede utilizar MT tanto para minimizar toxicidad como para maximizar la eficacia. Hoy en día, la rutina de medición de las concentraciones de agentes antimicrobianos está siendo una herramienta para mejorar el manejo clínico (Figura 1), como ejemplo de una MT asociada a la experiencia de cada centro hospitalario para generar un modelo poblacional en la pediatría. Los antimicrobianos para los que se realiza MT, hoy en día, son: aminoglucósidos, fármacos anti-retrovirales, triazoles 
y vancomicina ${ }^{2,3}$. El desafío de MT es la interpretación de las concentraciones medidas, para lo cual se requiere un equipo multidisciplinario incluyendo especialistas en farmacia clínica, microbiología y enfermedades infecciosas. Las decisiones sobre las recomendaciones de dosificación requieren una cuidadosa evaluación de criterios tales como: la dosis y el intervalo de administración, tiempo transcurrido desde el inicio del tratamiento, forma de administración del fármaco, duración total de la infusión para fármacos intravenosos, tiempo exacto de la extracción de sangre, edad, peso o superficie corporal del paciente, sus funciones renal y hepática, enfermedades subyacentes y la nutrición concomitante, el patógeno detectado o sospechado y su perfil de resistencia (idealmente CIMs) ${ }^{3}$.

Debido a la complejidad de poder realizar estudios clínicos en pacientes críticos, los estudios preclínicos in vitro y en animales, junto con modelos $\mathrm{FC} / \mathrm{FD}$, siguen siendo un soporte importante para la dosificación de fármacos en pacientes críticos con infecciones graves como se muestra en la Figura 2.

Llevamos a cabo una revisión no sistemática en

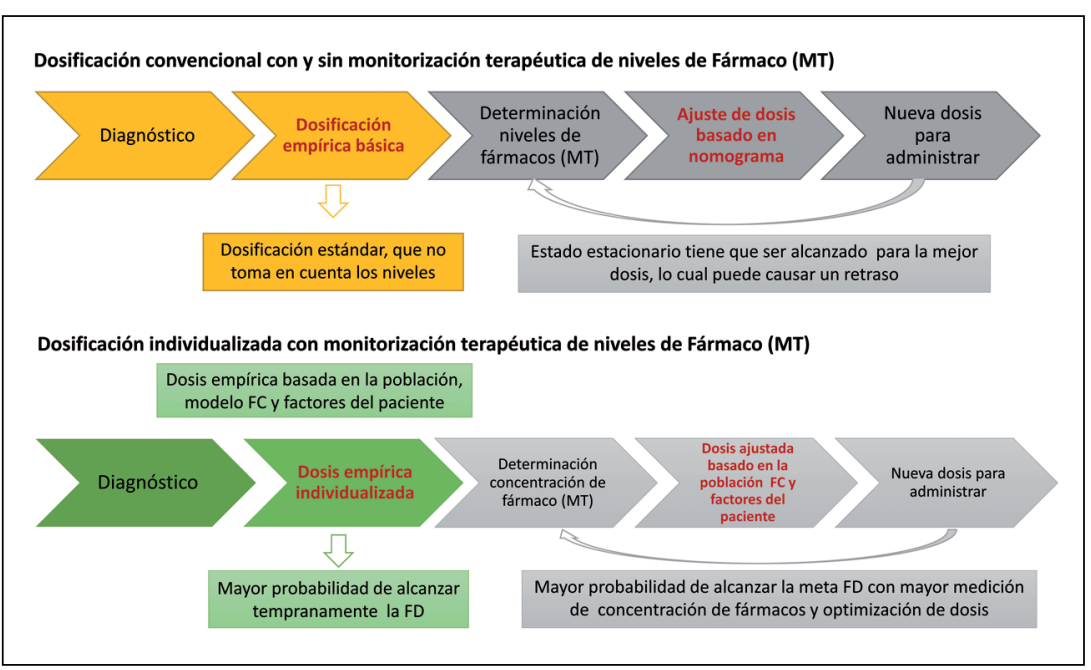

Figura 1. Superior: En amarillo dosificación antimicrobiana basada sólo en la información del producto. Inferior: La dosificación empírica se individualiza utilizando factores relevantes del paciente (por ejemplo, función renal o peso (verde) o donde la dosificación se basa en datos recogidos de la monitorización terapéutica (verde) y se adapta sobre la base de nomogramas de dosificación (arriba gris) o cálculo exacto de la FC del antimicrobiano individualizado para el paciente (abajo gris). (Adaptado de Tängdén et al ${ }^{30}$ ).

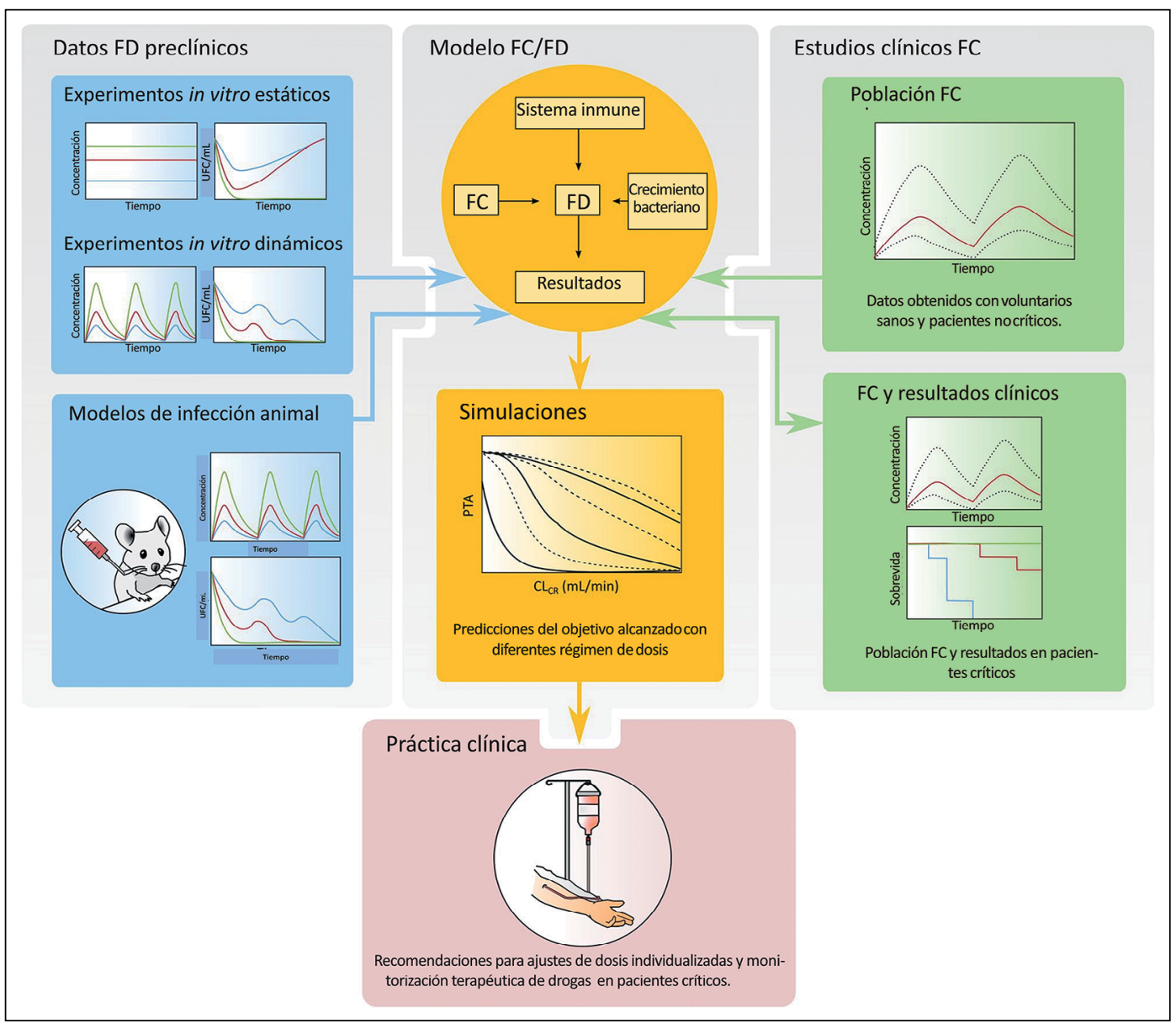

Figura 2. Rol de los modelos preclínicos de infección, modelos de FC/FD y estudios clínicos de FC para optimizar la dosificación de antimicrobianos y monitorización terapéutica en pacientes críticos con infecciones graves. Idealmente, los estudios futuros con este grupo de pacientes deberían evaluar las concentraciones de fármaco alcanzado en el sitio de la infección, además del plasma, y las asociaciones entre la FC medida y el resultado clínico. (Adaptado de Tängdén et al. ${ }^{30}$ ). 
PUBMED entre 2003 y 2016 sobre la evidencia científica de MT de antimicrobianos en pediatría, en los casos de vancomicina, amikacina y voriconazol, poniendo énfasis en cohortes latinoamericanas.

\section{Vancomicina}

Moise-Broder y cols ${ }^{4}$ describieron en adultos, el uso de $\mathrm{ABC} / \mathrm{CIM}$ para estimar el éxito clínico y microbiológico del tratamiento de la neumonía asociada a ventilación mecánica causada por Staphylococcus aureus, mostrando que un $\mathrm{ABC} / \mathrm{CIM}>345$ se relacionó con un mejor éxito clínico. Ello coincide con las actuales recomendaciones de la Sociedad Americana de Infectología que propone un $\mathrm{ABC} / \mathrm{CIM}>400$ como objetivo FC/FD 5 . Acuña y cols., encontraron que en 81 pacientes críticos pediátricos con dosis de $40 \mathrm{mg} / \mathrm{kg} /$ día, el parámetro objetivo ABC/CIM fue alcanzado sólo por $54 \%$ de la población estudiada, haciendo énfasis en la obtención de exposiciones sub óptimas con las dosis estudiadas de $40 \mathrm{mg} / \mathrm{kg} /$ día y la necesidad de adaptar los esquemas de dosis con criterios FC/FD, pudiendo establecer para la pediatría valores basales mayores a $10 \mu \mathrm{g} / \mathrm{mL}$ para obtener valores de $\mathrm{ABC} /$ $\mathrm{CIM}>400^{6}$. Villena y cols., evaluaron las concentraciones basales en pacientes pediátricos críticamente enfermos. Con dosis promedio de $47,1 \mathrm{mg} / \mathrm{kg} /$ día, obtuvieron en $60 \%$ de los casos basales menores a $10 \mu \mathrm{g} / \mathrm{mL}$ por lo que postulan iniciar tratamiento con dosis mayores y realizar monitoreo terapéutico de concentraciones plasmáticas en este grupo de pacientes ${ }^{7}$.

Giachietto y cols., estudiaron un grupo de 22 pacientes pediátricos críticos, con dosis de vancomicina de $40 \mathrm{mg} /$ $\mathrm{kg} /$ día; sólo $50 \%$ de los pacientes alcanzó ABC/CIM $>400$ considerando una CIM de $1 \mu \mathrm{g} / \mathrm{mL}$ para Staphylococcus aureus. Además, observaron que pacientes con balance positivo tenían Vd más altos y concentraciones más bajas que los pacientes con balance negativo, haciendo hincapié en el conocimiento del estado hídrico del paciente para la dosificación inicial del antimicrobiano ${ }^{8}$.

Zylbersztajn y cols., estudiaron un grupo de 51 pacientes pediátricos, con dosis iniciales de $40 \mathrm{mg} / \mathrm{kg}$ /día frac- cionadas cada $12 \mathrm{~h}$ o $60 \mathrm{mg} / \mathrm{kg}$ /día fraccionado cada $6 \mathrm{~h}$, según recomendaciones del vademecum de la institución, requiriendo aumento de dosis y frecuencia, mayormente en los esquemas de $40 \mathrm{mg} / \mathrm{kg} /$ día fraccionando cada 12 $\mathrm{h}$, llegando a obtener concentraciones terapéuticas (5 a $20 \mu \mathrm{g} / \mathrm{mL}$ ) con dosis de $40-50 \mathrm{mg} / \mathrm{kg} /$ día fraccionadas cada $6 \mathrm{~h}^{9}$ (Tabla 1$)$.

\section{Voriconazol}

Es un antifúngico triazólico utilizado comúnmente en el tratamiento de infecciones fúngicas invasoras (IFI) que afectan principalmente a pacientes inmunocomprometidos, como aspergilosis, candidiasis o aquellas producidas por Scedosporium sp y Fusarium sp, siendo el tratamiento de primera línea en aspergilosis invasora.

Voriconazol presenta una clara relación dosis respuesta, estrecho rango terapéutico y gran variabilidad FC, lo que hace imprescindible el MT de este fármaco. Por otra parte, se ha observado que existe una relación FC/FD para eficacia y toxicidad de este medicamento; por ejemplo, un nivel basal de voriconazol $<1 \mu \mathrm{g} / \mathrm{mL}$ se ha demostrado que se asocia con una mayor mortalidad infantil, mientras que las concentraciones superiores a $5,5 \mu \mathrm{g} / \mathrm{mL}$ se asociaron con aumento de la fototoxicidad y neurotoxicidad ${ }^{3}$. Por otra parte, Hyum y cols., demostraron en un estudio prospectivo que, a las 12 semanas de tratamiento, la tasa de fracaso terapéutico fue mayor en el grupo en que no se efectuó MT vs el que sí lo recibió en una cohorte poblacional pediátrica $(78,6$ vs. $40 \%)$, generando una evidencia clara del beneficio de efectuar $\mathrm{MT}^{10}$.

Existen variadas recomendaciones de dosificación de voriconazol en pediatría para el tratamiento de la aspergilosis invasora; la Sociedad Americana de Enfermedades Infecciosas (IDSA) recomienda de $5-7 \mathrm{mg} / \mathrm{kg} \mathrm{c} / 12 \mathrm{~h}$, la Asociación Americana de Pediatría, en el Red Book y la European Medicines Agency (EMA) recomiendan $8 \mathrm{mg} /$ $\mathrm{kg} \mathrm{c} / 12 \mathrm{~h}$. Sin embargo, se ha observado que $1 / 3$ de los pacientes entre 2-12 años pueden no lograr concentraciones adecuadas con las dosis anteriormente recomendadas ${ }^{12}$.

Varios estudios han demostrado que en muchos casos

\begin{tabular}{|c|c|c|c|c|c|c|}
\hline Referencia & n de pacientes & Dosis (mg/kg/día) & Vd (L/kg) & $T 1 / 2(h)$ & $\mathrm{Cl}(\mathrm{mL} / \mathrm{kg} / \mathrm{min})$ & $\begin{array}{c}\text { ABC/CIM } \\
\text { (CIM SA: } 1 \mu \mathrm{g} / \mathrm{mL} \text { ) }\end{array}$ \\
\hline Zylbersztajn ${ }^{9}$ (2008) & 51 & $50-60$ & $0,68(0,39-1,20)$ & $4,88(3,6-15,4)$ & & \\
\hline Giachetto $^{8}(2011)$ & 22 & 40 & $0,51 \pm 0,24$ & $3,1 \pm 0,78$ & $1,95 \pm 1,1$ & $364 \pm 218,9$ \\
\hline Acuña ${ }^{6}(2013)$ & 81 & 40 & $0,62(0,41-1,04)$ & $3,8(2,7-10,6)$ & & $410,6(261,5-689,1)$ \\
\hline Villena ${ }^{7}$ (2014) & 45 & 47 & $0,7(0,4-2,1)$ & $3,1(1,1-7,7)$ & & \\
\hline
\end{tabular}

Vd: volumen de distribución. T 1⁄2: vida media. Cl: clearance. ABC: área bajo la curva. CIM: concentración inhibitoria mínima. SA: Staphylococcus aureus. 
se necesitan dosis mayores para lograr concentraciones terapéuticas adecuadas. Neely y cols. ${ }^{13}$, sugieren que podría ser necesario dosis sobre $11 \mathrm{mg} / \mathrm{kg}$ cada $12 \mathrm{~h}$ para lograr concentraciones terapéuticas en niños. En una investigación de Hyum y cols., los pacientes requirieron altas dosis para mantener el objetivo terapéutico con una dosis promedio de $8,3 \mathrm{mg} / \mathrm{kg} /$ dosis en pacientes $<12$ años y $6,9 \mathrm{mg} / \mathrm{kg} / \mathrm{dosis}$ en $\geq 12$ años. Silva y cols., realizaron monitoreo terapéutico de voriconazol en pacientes oncológicos, observando que, para pacientes mayores de 12 años, dosis de 14-20 mg/kg/día pueden ser adecuadas para el tratamiento de aspergilosis invasora, pero en pacientes bajo 12 años de edad, se necesitarían dosis mayores, de hasta $30 \mathrm{mg} / \mathrm{kg} /$ día para alcanzar concentraciones basales mayores a $1,0 \mu \mathrm{g} / \mathrm{mL}$ en el grupo bajo dos años. Además, establecieron dosis mínimas de $400 \mathrm{mg} /$ día, vía oral, cada $8 \mathrm{~h}$, para tener concentraciones profilácticas mayores a $0,5 \mu \mathrm{g} / \mathrm{mL}$; además, las toxicidades encontradas no fueron relevantes frente al incremento de la dosis en pediatría ${ }^{14}$.

Por otra parte, es importante tener en consideración que la gran variabilidad interindividual en la exposición de voriconazol en niños y adolescentes hace que puedan tener alta o baja exposición con los regímenes propuestos. Por ejemplo, en el estudio de Friberg y cols., con regímenes de $8 \mathrm{mg} / \mathrm{kg}$ como dosis de mantenimiento, 10 a $20 \%$ de los niños presentaron sobreexposición y 10 a $20 \%$ subexposición ${ }^{15}$.

En pacientes adultos se ha descrito que la variabilidad intra-paciente e inter-paciente puede ser explicada por la cinética no lineal descrita para este fármaco, relacionada principalmente a la saturación del CYPC19 pero, su importancia clínica todavía no tiene evidencia sustentable para el ajuste de la dosificación. Sin embargo, varios estudios (Tabla 2) llevados a cabo en pacientes pediátricos inmunocomprometidos, de edades entre 2 y 12 años, encontraron que la no linealidad es menos pronunciada y la variabilidad interindividual parece ser mayor. Friberg y cols. ${ }^{15}$, usaron combinaciones de eliminación lineal y no lineal en su modelo con pacientes inmunocomprometidos pero notaron que la no linealidad FC de voriconazol fue menos pronunciada en niños entre 2-12 años de edad, quizás por su metabolismo de primer paso. Esto podría explicar, en parte, la necesidad de incrementar la dosis para lograr concentraciones plasmáticas terapéuticas ${ }^{12}$. Por otra parte, estudios han podido establecer que los niños tienen una gran capacidad por $\mathrm{kg}$ de peso para eliminar voriconazol comparado con la población adulta, pero a medida que se incrementan las dosis para obtener el intervalo terapéutico, se observan efectos del metabolismo saturable ${ }^{15}$.

Los parámetros FC/FD para voriconazol en la pediatría no han sido bien definidos. Buenos objetivos clínicos han sido correlacionados con la dosis total de voriconazol y el ABC/CIM en dosis fraccionada. En este contexto, la identificación de la especie proveería una indicación más precisa para una concentración objetivo, basada en la CIM del microorganismo, por ejemplo, en especies menos susceptibles como Candida krusei se necesitarían concentraciones basales alrededor de $5 \mu \mathrm{g} / \mathrm{mL}$; no obstante, es difícil este abordaje dada la baja sensibilidad de los cultivos ${ }^{12,15}$.

\begin{tabular}{|c|c|c|c|}
\hline Estudio & $\mathrm{N}$ de pacientes & Dosis inicial & $\begin{array}{l}\text { Dosis para lograr concentraciones } \\
\text { terapéuticas }\end{array}$ \\
\hline H M Kang et al ${ }^{10}(2015)$ & 61 (30 MT vs 31 No-MT) & $\begin{array}{l}\text { DC: } 6 \mathrm{mg} / \mathrm{kg} \mathrm{c} / 12 \mathrm{~h} \\
\text { DM: } 4 \mathrm{mg} / \mathrm{kg} \mathrm{c} / 12 \mathrm{~h}\end{array}$ & $\begin{array}{l}<12 \text { años: } 8,3 \mathrm{mg} / \mathrm{kg} \mathrm{c} / 12 \mathrm{~h} \\
\geq 12 \text { años: } 6,9 \mathrm{mg} / \mathrm{kg} \mathrm{c} / 12 \mathrm{~h}\end{array}$ \\
\hline Boast et al ${ }^{11}(2016)$ & 62 & IV: $7,1 \mathrm{mg} / \mathrm{kg} \mathrm{c} / 12 \mathrm{~h}$ & $\begin{array}{l}<6 \text { años: } 8,8 \mathrm{mg} / \mathrm{kg} \mathrm{c} / 12 \mathrm{~h} \\
6-12 \text { años: } 7,5 \mathrm{mg} / \mathrm{kg} \mathrm{c} / 12 \mathrm{~h} \\
>12 \text { años: } 4,0 \mathrm{mg} / \mathrm{kg} \mathrm{c} / 12 \mathrm{~h}\end{array}$ \\
\hline Friberg et al ${ }^{15}(2012)$ & $\begin{array}{l}112 \text { ( } 2-12 \text { años }) \\
26 \text { adolescentes ( } 12 \text { y }<17 \\
\text { años) y } 35 \text { adultos sanos }\end{array}$ & $\begin{array}{l}\text { Niños } \\
\text { IV: } 3-8 \mathrm{mg} / \mathrm{kg} / \text { día c/12 h } \\
\text { VO: } 4-6 \text { y } 200 \mathrm{mg} \mathrm{c} / 12 \mathrm{~h} \\
\text { Adultos y adolescentes } \\
\text { IV: } 6 \mathrm{mg} / \mathrm{kg} / \text { dosis } \mathrm{c} / 12 \mathrm{~h} \text { seguido de } 4 \mathrm{mg} / \mathrm{kg} / \text { dosis } \mathrm{c} / 12 \mathrm{~h} \\
\text { VO: } 300 \mathrm{mg} \mathrm{c} / 12 \mathrm{~h}\end{array}$ & $\begin{array}{l}\text { Niños } \\
\text { IV: DC: } 9 \mathrm{mg} / \mathrm{kg} \mathrm{c} / 12 \mathrm{~h} \\
\text { DM: } 4 \text { y } 8 \mathrm{mg} / \mathrm{kg} \mathrm{c} / 12 \mathrm{~h} \\
\text { VO: } 9 \mathrm{mg} / \mathrm{kg} \mathrm{c} / 12 \mathrm{~h} \text { (máx } 350 \mathrm{mg} \mathrm{c} / 12 \mathrm{~h} \text { ) }\end{array}$ \\
\hline Silva et $\mathrm{al}^{14}(2016)$ & 26 & $\begin{array}{l}\text { IV: } 14 \mathrm{mg} / \mathrm{kg} / \mathrm{día} \\
\text { VO: } 200 \mathrm{mg} \mathrm{c} / 12 \mathrm{~h}\end{array}$ & $\begin{aligned} \mathrm{IV}: & \geq 12 \text { años: } 14-20 \mathrm{mg} / \mathrm{kg} / \mathrm{día} \\
& <12 \text { años: } 8-30 \mathrm{mg} / \mathrm{kg} / \mathrm{dí} a \\
\text { VO: } & 200 \mathrm{mg} \mathrm{c} / 12 \mathrm{~h}\end{aligned}$ \\
\hline
\end{tabular}

MT: monitoreo terapéutico. DC: dosis de carga. DM: dosis de mantenimiento. IV: intravenoso. VO: vía oral. 


\begin{tabular}{|c|c|c|c|c|c|c|}
\hline Referencia & n pacientes & Dosis (mg/kg/día) & Vd (Lt/kg) & $T^{1 / 2}(\mathbf{h})$ & $\mathrm{Cl} \mathrm{Cr}(\mathrm{mL} / \mathrm{min})$ & $C \max (\mu \mathrm{g} / \mathrm{mL})$ \\
\hline $\begin{array}{l}\text { Rabello M² } \\
\text { (2011) }\end{array}$ & 35 & $\begin{array}{l}\text { DM:7,5 mg/kg/dosis } \\
\text { DD:15 mg/kg/día }\end{array}$ & $\begin{array}{l}\text { DM: } 0,64 \pm 0,47 \\
\text { DD: } 0,55 \pm 0,23\end{array}$ & $\begin{array}{l}\text { DM: } 2,94 \pm 0,3 \\
\text { DD: } 8,05 \pm 1,5\end{array}$ & $\begin{array}{c}\text { DM: } 83,5 \pm 7,2 \\
\text { DD: } 36,33 \pm 4,5\end{array}$ & $\begin{array}{l}\text { DM } 14,42 \pm 3,27 \\
\text { DD: } 27,19 \pm 2,43\end{array}$ \\
\hline $\begin{array}{l}\text { Belfayol L28 } \\
(1991)\end{array}$ & 35 & $15 \mathrm{mg} / \mathrm{kg} / \mathrm{día}$ & $\begin{array}{l}0,36 \\
\pm 0,08\end{array}$ & $\begin{array}{l}2,0 \\
\pm 1,0\end{array}$ & $\begin{array}{l}56,3 \\
\pm 33,8\end{array}$ & $\begin{array}{l}31,3 \pm 59,0 \text { (día 2) } \\
32,4 \pm 17,4 \text { (día 5) }\end{array}$ \\
\hline $\begin{array}{l}\text { Rodríguez JC29 } \\
\text { (2003) }\end{array}$ & 15 & DM 10,61 $\pm 1,78(c / 8)$ & $0,37 \pm 0,12$ & $1,75 \pm 0,29$ & - & $29,12 \pm 7,65$ \\
\hline
\end{tabular}

\begin{abstract}
Amikacina
Amikacina es el aminoglucósido más utilizado para infecciones graves causadas por bacilos gramnegativos $^{16,17}$. Dentro de sus características se destaca un efecto bactericida rápido y sinergia cuando se usa junto a $\beta$-lactámicos ${ }^{18,19}$. Su mecanismo de acción tiene dos fases, la primera de efecto bactericida rápido, que depende de la concentración máxima, y la segunda fase de efecto bactericida lento, que es independiente de la concentración del fármaco, y conocida como efecto post antibiótico, base para recomendar su uso como monodosis diaria ${ }^{20-23}$. El uso de monodosis de amikacina en el tratamiento de
\end{abstract}

neutropenia febril en adultos y niños es reconocido en la literatura médica ${ }^{27-29}$ (Tabla 3), habiéndose demostrado su eficacia clínica y microbiológica. La máxima respuesta clínica ocurre cuando las concentraciones plasmáticas máximas son mayores que la CIM por un factor de al menos 8 en niños.

Tréluyer y cols., analizaron el uso de dosis únicas y múltiples diarias de amikacina en 155 neonatos, lactantes y niños a través de monitorización farmacocinética, recomendando el uso de monodosis en todas las edades y una Cmáx superior a la CIM por un factor de al menos 8 , con una concentración basal menor que $1 \mu \mathrm{g} / \mathrm{mL}^{21-29}$.

En un estudio realizado en pacientes pediátricos con

\section{Tabla 4. Sugerencias de ajuste de dosis para antimicrobianos asociados a concentraciones de fármacos}

\section{Fármacos Control de concentraciones de fármacos}

Vancomicina Concentraciones basales $(5-15 \mu \mathrm{g} / \mathrm{mL}) 30$ min previo a la administración de la próxima dosis. Concentraciones óptimas para $S$. aureus $\mathrm{CIM}=1 \geq 10 \mu \mathrm{g} /$ $\mathrm{mL}$ equivalente a $\mathrm{ABC} / \mathrm{CIM}>400$

Concentraciones pico $(25-45 \mu \mathrm{g} / \mathrm{mL}) 30$ min post administración. Concentraciones óptimas para $S$. aureus $\mathrm{CIM}=1 \geq 28 \mu \mathrm{g} / \mathrm{mL}$ equivalente a $\mathrm{ABC}$ / $\mathrm{CIM}>400$

En pacientes con Crs $<<0,2 \mu \mathrm{g} / \mathrm{dL}$ evaluar prolongación de infusión de administración de 2-3 h esperando concentraciones basales $>10 \mu \mathrm{g} / \mathrm{mL}$

Amikacina Concentraciones basales (DD: $0-2 \mu \mathrm{g} / \mathrm{mL} \mathrm{DM}:<10 \mu \mathrm{g} / \mathrm{mL}$ ) $30 \mathrm{~min}$ previo a la administración de la próxima dosis Concentraciones pico $(20-64 \mu \mathrm{g} / \mathrm{mL}) 30$ min post término de la infusión En pacientes con $\mathrm{Crs}<<0,2 \mu \mathrm{g} / \mathrm{mL}$, fibrosis quística, shock séptico o gran quemado, evaluar uso de dosis mayores

Voriconazol Concentraciones basales $(1-5,5 \mu \mathrm{g} / \mathrm{mL}) 30$ min previo a la administración de la próxima dosis. Para aspergilosis invasora se sugiere concentraciones basales $>2 \mu \mathrm{g} / \mathrm{mL}$ en tratamiento

Concentraciones basales para profilaxis son: $>0,5 \mu \mathrm{g} / \mathrm{mL}$. Control de concentraciones asociado a efectividad de tratamiento

Concentraciones pico $(6-9 \mu \mathrm{g} / \mathrm{mL}) 1 \mathrm{~h}$ post administración. Concentraciones asociado al control de toxicidad

\section{Sugerencia de ajuste}

Pacientes con $S$. aureus $\mathrm{CIM}=1 \mu \mathrm{g} / \mathrm{mL}$, se debe ajustar dosis si no alcanzan concentraciones objetivo, desde 60 a $95 \mathrm{mg} / \mathrm{kg} /$ día equivalente al modelo farmacométrico de $\mathrm{ABC} / \mathrm{CIM}>400$, monitorizando función renal e interacciones con otros agentes nefrotóxicos, revisar ajuste si aparece otro microorganismo

Evaluar uso de infusión continua para vancomicina en pacientes de neonatología con monitorización de la concentración en estado estacionario

Ajuste de dosis asociada al aislamiento microbiológico teniendo en cuenta que su modelo farmacométrico es concentración dependiente $\mathrm{Cmax} / \mathrm{CIM}$ $>8$ para bacilos gramnegativos. Las dosis pueden ajustarse entre $15-35 \mathrm{mg} /$ $\mathrm{kg}$ controlando la función renal y concentraciones plasmáticas. Se recomienda una dosis diaria. El monitoreo de concentración pico es una medición para asegurar efectividad

Las dosis intravenosas iniciales en pediatría para sospecha de aspergilosis bajo 12 años de edad deben ser de 16-18 mg/kg/día, fraccionado cada $8 \mathrm{~h}$ y control de concentraciones plasmáticas a las $96-120 \mathrm{~h}$ para obtener concentraciones óptimas de efectividad

Para los tratamientos vía oral la sugerencia también es poder ajustar a cada $8 \mathrm{~h}$ para alcanzar concentraciones óptimas de tratamiento y/o profilaxis

ABC: área bajo la curva. CIM: concentración inhibitoria mínima. Crs: creatinina sérica. Cmax: concentración máxima. DM: dosis múltiples. DD: dosis diaria. 
neutropenia febril post-quimioterapia, Rabello y cols., evidenciaron que los niveles pico alcanzados con dosis de $15 \mathrm{mg} / \mathrm{kg}$ /día cada $24 \mathrm{~h}$ fueron suficientes en $30 \%$ de los pacientes estudiados, mientras que en los pacientes que recibieron la misma dosis fraccionada en dos veces al día, sólo 8\% alcanzó niveles pico terapéuticos, mostrando la necesidad de monitorizar las concentraciones plasmáticas para lograr un ajuste individualizado de los esquemas de dosificación. Los datos se presentan en la Tabla 3 y la sugerencia de dosificar una vez al día, de acuerdo a modelo concentración dependiente, juega un rol fundamental ${ }^{27}$.

Finalmente, confeccionamos una sugerencia de dosis iniciales y MT basada en la literatura científica analizada en el presente trabajo sobre los tratamientos con vancomicina, amikacina y voriconazol en pediatría en la Tabla 4.

\section{Conclusión}

La importancia de controlar concentraciones plasmáticas de fármacos en pediatría es fundamental para optimizar los tratamientos antimicrobianos y asegura una mayor efectividad y seguridad de los medicamentos utilizados.
Es importante mencionar que se deben incrementar los estudios y experiencias en el uso de antimicrobianos con monitorización terapéutica, para asegurar una dosis más cercana a los modelos poblacionales pediátricos.

\section{Resumen}

La presente revisión resume la evidencia sobre la monitorización terapéutica de tres antimicrobianos basada en datos regionales: vancomicina, amikacina y voriconazol en la población pediátrica. Estos datos coinciden con la literatura internacional en relación al requerimiento de dosis mayores que $40 \mathrm{mg} / \mathrm{kg} /$ día de vancomicina, la posibilidad de usar monodosis diarias de amikacina y el requerimiento de dosis mayores de voriconazol en relación a las iniciales recomendadas de $8 \mathrm{mg} / \mathrm{kg} / \mathrm{día}$. Contar con datos locales sobre el comportamiento farmacocinético/farmacodinámico de diversos antimicrobianos en la pediatría es de gran valor para adecuar la dosificación de los mismos en nuestra población. Se deberían incrementar los estudios de monitorización terapéutica en el uso de antimicrobianos en pediatría que permitan generar pautas de tratamiento adecuadas para este grupo etario.

\section{Referencias bibliográficas}

1.- Kimland E, Odlind V. Off-label drug use in pediatric patients. Clin Pharmacol Ther 2012; 91: 796-801.

2.- Kearns G L, Abdel-Rahman S M, Alander S W, Blowey D L, Leeder J S, Kauffman R E. Developmental pharmacology-drug disposition, action and therapy in infants and children. $\mathrm{N}$ Engl J Med 2003; 349 (12): 1157-67.

3.- Ritz N, Bielicki J, Pfister M, van der Anker J. Therapeutic drug monitoring for anti-infective agents in pediatrics. The way forward. Pediatr Infect Dis J. 2016; 35 (5): 570-2.

4.- Moise-Broder P, Sakoulas G, Eliopoulos G M, Schentag J J, Forrest A, Moellering $\mathrm{R} C \mathrm{Jr}$. Accessory gene regulator group II polymorphism in methicillin resistant Staphylococcus aureus is predictive of failure of vancomycin therapy. Clin Infect Dis 2004; 38: 1700-5.

5.- Liu C, Bayer A, Cosgrove S E, Daum R S, Fridkin S K, Gorwitz R J, et al. Clinical practice guidelines by the Infectious Diseases Society of America for the treatment of methicillin-resistant Staphylococcus aureus infections in adults and children. Clin Infect Dis 2011; 52 (3): e18-55. Erratum in: Clin Infect Dis 2011; 53 (3): 319.

6.- Acuña C, Morales J, Castillo C, Torres J P. Farmacocinética de vancomicina en niños hospitalizados en una unidad de paciente crítico. Rev Chilena Infectol 2013; 30 (6): 585 90.

7.- Villena R, González C, Nalegach M E, Vásquez A, Villareal M, Drago M. Monitoreo terapéutico de vancomicina intravenosa en una unidad de paciente crítico pediátrico. Rev Chilena Infectol 2014; 31 (3): 249-53.

8.- Giachetto G A, Telechea H M, Speranza N, Oyarzun M, Nanni L, Menchaca A. Vancomycin pharmacokineticpharmacodynamic parameters to optimize dosage administration in critically ill children. Pediatr Crit Care Med 2011; 12 (6): e250-4.

9.- Zylbersztajn B L, Travaglianti M, Weller G, Mato H G. Therapeutic monitoring of vancomycin in critical care pediatric patients. Arch Argent Pediatr 2008; 106 (1): 26-31. Erratum in: Arch Argent Pediatr 2008; 106 (2): 175.

10.- Hyun Mi Kang, Hoan Jong Lee, Eun Young Cho, Kyung-Sang Yu, Hyunju Lee, Ji Won Lee, et al. The clinical significance of voriconazole therapeutic drug monitoring in children with invasive fungal infections. Pediatr Hematol Oncol 2015; 32: 1-11.

11.- Boast A, Curtis N, Cranswick N, Gwee A. Voriconazole dosing and therapeutic drug monitoring in children: experience from a paediatric tertiary care centre. J Antimicrob Chemother 2016; 71 (7): 2031-6.
12.- Job K, Jared Olson, Stockmann C, Constance J, Enioutina E, Rower J, et al. Pharmacodynamic studies of voriconazole: informing the clinical management of invasive fungal infections. Expert Rev Anti Infect Ther 2016; 14 (8): 731-46.

13.- Neely M, Margol A, Fu X, van Guilder M, Bayard D, Schumitzky A, et al. Achieving target voriconazole concentrations more accurately in children and adolescents. Antimicrob Agents Chemother 2015; 59: 3090-7.

14.- Silva F, Navea D, Salas C, Torres J P, Catalán P, Morales J. Analysis of voriconazole serum concentrations and safety profile in pediatric oncology patients. Rev Chilena Infectol 2016; 33 (2): 127-34.

15.- Friberg L, Ravva P, Karlsson M, Liuc P. Integrated population pharmacokinetic analysis of voriconazole in children, adolescents, and adults. Antimicrob Agents Chemother 2012; 56 (6): 3032-42.

16.- Hoban D, Bouchillon S, Hawser S, Badal R, Labombardi V, DiPersio J. Susceptibility of gram-negative pathogens isolated from patients with complicated intraabdominal infections in the United States, 2007-2008: Results of the Study for Monitoring Antimicrobial Resistance Trends (SMART). Antimicrob Agents Chemother 2010; 54 (7): 3031-4. 
17.- Nicoletti G, Schito G, Fadda G, Boros S, Nicolosi D, Marchese A, et al. Bacterial isolates from severe infections and their antibiotic susceptibility patterns in Italy: A nationwide study in the hospital setting. J Chemother 2006; 18 (6): 589-602.

18.- Charnas R, Luthi A R, Ruch W. Once daily ceftriaxone plus amikacin $v s$ three times daily ceftazidime plus amikacin for treatment of febrile neutropenic children with cancer. Pediatr Infect Dis J 1997; 16 (4): 346-53.

19.- Bartal C, Danon A, Schlaeffer F. Pharmacokinetic dosing of aminoglycosides: a controlled trial. Am J Med 2003; 114 194-8.

20.- Marik P E, Lipman J, Kobilski A, Scribante J. A prospective randomized study comparing once $v s$ twice-daily amikacin dosing in critically ill adult and pediatric patients. J Antimicrob Chemother 1991; 28: 753-64.

21.- Tréluyer J M, Merlé Y, Tonnelier S, Rey E, Pons G. Nonparametric population pharmacokinetic analysis of amikacin in neonates, infants, and children. Antimicrob
Agents Chemother 2002; 46 (5): 1381-7.

22.- Shahid M, Cooke R. Is a once daily dose of gentamicin safe and effective in the treatment of UTI in infants and children? Arch Dis Child 2007; 92 (9): 823-4.

23.- Jamshaid M, Yousuf S, Bukhari N I, Rizvi A A. Relative pharmacokinetics of three amikacin brands in onco-hemotologic pediatric patients experiencing febrile neutropenia. Eur J Drug Metab Pharmacokinet 2003; 28 (1): 1-6.

24.- Carpetis J, Jaquiery A, Buttery J. Randomized, controlled trial comparing once daily and three times daily gentamicin in children with urinary tract infections. Pediatr Infect Dis J 2001; 20: 240-6.

25.- Kraus D, Pai M, Rodvold K. Efficacy and tolerability of extended interval aminoglycoside administration in pediatric patients. Pediatr Drugs 2002; 4 (7): 469-84.

26.- Contopoulos-Ioannidis D, Giotis N, Baliatsa D, Ioannidis J. Extended-interval aminoglycoside administration for children: a meta-analysis. Pediatrics 2004; 114: e111-8.

27.- Rabello M, Villena R, Morales J, Aravena R,
Kopp K, Villarroel M, et al. Pharmacokinetics of amikacin in children with cancer and febrile neutropenia. Poster, 49th Annual Meeting of the Infectious Diseases Society of America (IDSA), October 20-23, 2011. https://www.researchgate. net/publication/267912421_Pharmacokinetics of_amikacin_in_children_with_cancer_and febrile_neutropenia.

28.- Belfayol L, Talon P, Eveillardl M, Alet P, Fauvelle F. Pharmacokinetics of once-daily amikacin in pediatric patients Clin Microbiol Infect 1996; 2 (3): 186-91.

29.- Rodríguez J C, Schoenike S, Scott G, RossiqueGonzález M T, Gómez-Marín O. An evaluation of gentamicin, tobramycin, and amikacin pharmacokinetic/pharmacodynamic parameters in HIV-infected children. J Pediatr Pharmacol Ther 2003; 8: 274-82.

30.- Tängdén T, Ramos M V, Felton T W, Nielsen E I, Marchand S, Brüggemann R J, et al. The role of infection models and PK/PD modelling for optimizing care of critically ill patients with severe infections. Intensive Care Med 2017; 43 : $1-12$ 